\title{
Häufigkeiten von Übergriffen auf Kinder und Jugendliche durch Angehörige der Heil- und Pflegeberufe
}

\author{
Miriam Rassenhofer, Simone Korger, Jörg M. Fegert und Ulrike Hoffmann
}

Klinik für Kinder- und Jugendpsychiatrie/Psychotherapie, Universitätsklinikum Ulm

\begin{abstract}
Zusammenfassung: Theoretischer Hintergrund: Kindesmisshandlung, -missbrauch und Vernachlässigung stellen ein gesamtgesellschaftliches Entwicklungsrisiko für Kinder in Deutschland dar. Zweithäufigster Kontext des Geschehens nach dem familiären Umfeld sind Institutionen. Fragestellung: Der vorliegende Artikel gibt eine Übersicht zu Häufigkeiten von Übergriffen durch Angehörige der Heil- und Pflegeberufe mit Schwerpunkt auf dem kinder- und jugendpsychiatrischen/-psychotherapeutischen Bereich. Methode: Mittels Literaturrecherche wird der Forschungsstand zum Thema dargestellt. Ergebnisse: Neben Auswertungen zu Zwangsmaßnahmen, welche von Betroffenen häufig als viktimisierend erlebt werden, existieren für Deutschland zwei repräsentative retrospektive Befragungen zum medizinischen Bereich. Hier ergaben sich Prävalenzen zwischen einem Fünftel und einem Drittel der Befragten, die mindestens eine Form von Gewalt oder Vernachlässigung bejahten. Diskussion und Schlussfolgerung: Neben alarmierenden Zahlen zeigt sich der große Bedarf an weiterer Forschung zum Thema.
\end{abstract}

Schlüsselwörter: Kindesmisshandlung, Vernachlässigung, institutioneller Kindesmissbrauch, medizinisch-therapeutisches Personal, Zwangsmaßnahmen, medizinische Einrichtungen

\begin{abstract}
Prevalence of Child Abuse and Neglect by Health Professionals
Abstract: Background: Child abuse and neglect pose a permanent developmental risk for children and adolescents. After the family environment, the second most frequent context in which abuse occurs is institutions. However, institutions in the medical and psychotherapeutic fields have rarely been the subject of epidemiological studies, despite the existing institutional and individual risk factors, like stressful working conditions or patients' previous experiences of child maltreatment. Besides physical, emotional, and sexual abuse as well as neglect, in the medical and therapeutical context, there is also the risk that children experience coercive measures that may be perceived as traumatizing and victimizing even if they're medically justified or necessary. Objective: This article gives an overview of the research on the prevalence rates of child abuse, neglect, and coercive measures perpetrated by health professionals, focusing especially on the field of child and adolescent psychiatry and psychotherapy. Method: Using a keyword-based literature search, we identified studies reporting prevalences of child maltreatment perpetrated by health professionals in medical-therapeutic institutions, general and pediatric hospitals, departments of child and adolescent psychiatry and psychotherapy, in addition to epidemiological studies on adult patients. Results: Apart from analyses concerning the frequency of coercive measures, which were experienced by $10-25 \%$ of child and adolescent psychiatric inpatients, there exist two representative retrospective surveys on the German population, one of which differentiates between distinct types of medical-therapeutic institutions. In the latter, between one-fifth and one-third of the participants reported having experienced at least one type of abuse or maltreatment during hospitalization in their childhood, whereby the prevalences in departments for child and adolescent psychiatry/psychotherapy were always higher than in general and pediatric hospitals. Physical abuse (10-31\%), emotional abuse $(10-23 \%)$, and neglect $(12-22 \%)$ were the most frequent forms experienced by the respondents. Child sexual abuse by health professionals was less frequent, with $0.8 \%$ of the participants reporting incidents in medical-therapeutic institutions in general, whereas there was a much higher prevalence of $7.3 \%$ in child and adolescent psychiatry. Discussion and Conclusion: This article gives an overview of the prevalence of child maltreatment in the medical and therapeutic fields. Because of the lack of epidemiological studies, we included inquiries on different samples and of varying methodological quality. Therefore, the article does not represent a systematic overview but rather provides a first estimation of the problem. Besides indicating alarmingly high prevalence rates, it also shows the need for further research on the issue.
\end{abstract}

Keywords: child maltreatment, neglect, institutional child abuse, health professionals, maltreatment, coercive measures, medicaltherapeutic institutions

Kindesmisshandlung stellt ein gesamtgesellschaftliches Problem dar. In Deutschland gab in retrospektiven Repräsenta- tivbefragungen etwa eine von drei Personen an, in ihrer Kindheit von mindestens einer Form der Gewalt oder Ver- 
nachlässigung betroffen gewesen zu sein (Iffland, Brähler, Neuner, Häuser \& Glaesmer, 2013; Witt, Brown, Plener, Brähler \& Fegert, 2017; Witt, Glaesmer et al., 2018). In der im Jahr 2016 befragten repräsentativen Stichprobe bejahten jeweils zwischen 6 und $8 \%$ der Teilnehmenden mindestens als mäßig wahrgenommene Erlebnisse körperlicher, emotionaler und sexualisierter Gewalt in ihrer Kindheit. Von emotionaler und körperlicher Vernachlässigung waren mit Prävalenzen von $13 \%$ und $23 \%$ noch größere Anteile der Bevölkerung betroffen (Witt et al., 2017). Dabei scheint es zwar Kohortenunterschiede bezüglich der Form der erlebten Gewalt zu geben, etwa die erhöhte Prävalenz körperlicher Misshandlung und Vernachlässigung in älteren Kohorten (Iffland et al., 2013; Witt et al., 2017), jedoch keine generelle Abnahme der Gesamtprävalenz von Misshandlung und Vernachlässigung, was durch den Vergleich zweier Querschnittstichproben gezeigt werden konnte (Witt, Glaesmer et al., 2018). Daraus lässt sich schließen, dass Misshandlung, Missbrauch und Vernachlässigung in Deutschland nicht nur ähnlich häufig vorkommen wie in anderen Ländern der Welt (vgl. Rassenhofer, 2020), sondern ein dauerhaftes Risiko für Kinder und Jugendliche darstellen.

Kinder und Jugendliche erfahren Misshandlung, Missbrauch und Vernachlässigung häufig im Kontext ihrer Familie, jedoch zeigen Forschungsergebnisse insbesondere aus dem letzten Jahrzehnt, dass auch in Einrichtungen, in denen Kinder und Jugendliche leben oder betreut werden, Übergriffe kein vernachlässigbares Problem sind (vgl. SpröberKolb et al., 2017). Im folgenden werden unter den Begriff Übergriff sowohl körperliche, emotionale und sexualisierte Gewalt als auch körperliche und emotionale Vernachlässigung oder Zwangsmaßnahmen wie Fixierung, Isolation und Zwangsmedikation gefasst. Während die negativen Auswirkungen von Kindesmisshandlung bereits vielfach belegt wurden (vgl. z.B. die Übersicht von Schulte, Szota \& Christiansen, 2021), konnten neuere Studien nachweisen, dass Zwangsmaßnahmen, auch dann wenn sie notwendig und (medizinisch) indiziert sind, von den Patient_innen und ihren Eltern ebenfalls als traumatisierend oder viktimisierend wahrgenommen werden können (Aguilera-Serrano, Guzman-Parra, Garcia-Sanchez, Moreno-Küstner \& MayoralCleries, 2018; Hoffmann, 2020). In einer bevölkerungsrepräsentativen Studie lag die Prävalenz sexuellen Kindesmissbrauchs in Institutionen in Deutschland bei $3.1 \%$, wobei Frauen (4.8\%) deutlich häufiger betroffen waren als Männer $(0.8 \%)$. Noch häufiger waren Erlebnisse körperlicher Gewalt in einer Institution, welche von $28 \%$ der befragten Personen berichtet wurden. Etwa die Hälfte der sexuellen und körperlichen Übergriffe ging dabei von Personal aus (Witt, Rassenhofer et al., 2019).

Dass Kinder in Einrichtungen, die ihrer Versorgung, Erziehung und Pflege dienen sollen, Gewalt, Vernachlässigung oder Zwangsmaßnahmen erfahren, lässt sich durch ein Zusammenspiel verschiedener Risikofaktoren erklären (siehe auch Hoffmann, Fegert, König, Maier \& Herberhold, 2021). Auf der organisatorischen Ebene solcher Einrichtungen können etwa stark hierarchische Strukturen zu einem Machtungleichgewicht führen, aufgrund dessen Kinder und Jugendliche sich kaum wehren können und ihnen, selbst wenn sie sich offenbaren, häufig nicht geglaubt und entsprechend geholfen wird, da die Täter_innen einen höheren Status haben (Spröber et al., 2014; Spröber-Kolb et al., 2017). Auf Personalebene sind beispielsweise hohe Arbeitsbelastung, zu geringe Personalschlüssel, positive Einstellung zu körperlicher Bestrafung, distanzierte Pflegeverhältnisse oder Wunsch nach Normalität Risikofaktoren für den Einsatz von Gewalt oder Zwangsmaßnahmen zu nennen (siehe Hoffmann et al., 2021; Roy et al., 2021). Schließlich belegen Studien auch Vulnerabilitäten auf individueller Ebene. Hierzu zählen unter anderem zurückliegende Gewalt- und Vernachlässigungserfahrungen in der Herkunftsfamilie (Katz, Courtney \& Novotny, 2017), emotionale und Verhaltensauffälligkeiten sowie das Geschlecht (Roy et al., 2021). Generell sind laut Dunkelfelduntersuchungen Mädchen häufiger von sexuellem Missbrauch und emotionaler Misshandlung betroffen als Jungen (Iffland et al., 2013; Stoltenborgh, van IJzendoorn, Euser \& BakermansKranenburg, 2011; Witt et al., 2017). Kinder mit Behinderung haben ein drei- bis fünffach erhöhtes Risiko, sexualisierter, körperlicher und emotionaler Gewalt oder Vernachlässigung ausgesetzt zu sein (Jones et al., 2012).

Obwohl viele dieser Risikofaktoren auch Kinder betreffen, die medizinisch, psychiatrisch und psychotherapeutisch betreut werden, gibt es bislang nur wenige Studien, welche die Prävalenz von Übergriffen in diesen Bereichen systematisch untersuchen (Spröber-Kolb et al., 2017). Ziel dieses Übersichtsartikels ist es, den aktuellen Forschungsstand $\mathrm{zu}$ den Häufigkeiten von Übergriffen durch Angehörige der Heil- und Pflegeberufe wiederzugeben.

Hierfür wurden mittels Schlüsselwortsuche in elektronischen Zeitschriftendatenbanken (Web of Science, PubMed) identifizierte deutsch- und englischsprachige Fachartikel zur Prävalenz körperlicher, emotionaler und sexualisierter Gewalt, Vernachlässigung sowie Zwangsmaßnahmen im Rahmen allgemeinmedizinischer, pädiatrischer sowie kinder- und jugendpsychiatrischer/-psychotherapeutischer Behandlung berücksichtigt, die sich auf Übergriffe durch medizinisch-therapeutisches Personal auf Kinder und Jugendliche beziehen. Zusätzlich wurden nach dem Schneeballprinzip relevante Quellen der identifizierten Artikel über google scholar gesucht. Aufgrund der geringen Studienlage wurden teilweise ergänzend Untersuchungen zu Prävalenzen von Übergriffen auf erwachsene Patient_innen herangezogen. 


\section{Häufigkeit von Übergriffen in medizinischen Institutionen}

\section{Daten aus Nordeuropa}

Studien zur Häufigkeit von Misshandlung im medizinischen Kontext wurden bislang überwiegend im nordeuropäischen Raum, genauer in Schweden, Norwegen, Dänemark, Island, Belgien, Finnland und Estland, durchgeführt (Lukasse et al., 2015; Swahnberg et al., 2007; Swahnberg, Davidsson-Simmons, Hearn \& Wijma, 2012). Das in allen diesen Studien verwendete NorVold Abuse Questionnaire (NorAQ) erfragt bisherige Erlebnisse von Misshandlung im Rahmen medizinischer Behandlungen sowie andere Erlebnisse emotionaler, körperlicher und sexueller Gewalt (Swahnberg \& Wijma, 2003). Während der Großteil der Misshandlungserlebnisse studienübergreifend im Erwachsenenalter geschehen war, bejahten in einer ersten Studie 1.0 \% der befragten Frauen Erlebnisse leichter, $1.7 \%$ Erlebnisse moderater und 1.2\% Erlebnisse schwerer Misshandlung bei medizinischen Behandlungen im Kindesalter (Swahnberg et al., 2007). Von mindestens einer Misshandlungserfahrung bei medizinischen Behandlungen im Kindesalter berichteten $4.1 \%$ der Frauen und $1.8 \%$ der Männer (Lukasse et al., 2015; Swahnberg et al., 2012). Es konnte zudem gezeigt werden, dass das Risiko hierfür bei Personen, die zuvor schon einmal von Gewalt betroffen waren, in etwa doppelt so hoch ist (Swahnberg et al., 2007).

\section{Daten aus Deutschland}

In Deutschland gibt es neben Einzelfallberichten bislang kaum Erhebungen zur Prävalenz von Kindesmisshandlung und Vernachlässigung im medizinischen Bereich (Hoffmann, Fegert, Jud, Clemens \& Rassenhofer, 2021). Erste Anhaltspunkte auf Übergriffe durch medizinisches Personal in Deutschland stammen aus der Begleitforschung zur Telefonischen Anlaufstelle der ersten unabhängigen Beauftragten zur Aufarbeitung des sexuellen Kindesmissbrauchs (UBSKM). Obwohl als Reaktion auf die 2010 bekannt gewordenen Missbrauchsfälle in erzieherischen und kirchlichen Institutionen ins Leben gerufen, waren unter den 4570 anrufenden Betroffenen 59 Personen (ca. 1\% der Betroffenen), die im Rahmen medizinischer oder psychotherapeutischer Behandlungen sexuell missbraucht wurden (Fegert, Bergmann, Spröber \& Rassenhofer, 2013). Häufiger als in der Gesamtstichprobe bestand bei diesen geschilderten Übergriffen, die zu $94 \%$ von männlichen Behandlern ausgingen, in 40 \% der Fälle kein Körperkontakt zwischen Täter und Betroffenen. In $27 \%$ der Fälle kam es zur Penetration.
Die Betroffenen berichteten, dass ihnen aufgrund der gesellschaftlich angesehenen Stellung der Behandelnden häufig nicht geglaubt worden war, wenn sie Bezugspersonen von der Tat erzählten.

In einer ersten Repräsentativbefragung zur Prävalenz von sexuellem Kindesmissbrauch in Einrichtungen in Deutschland gaben $0.1 \%$ der befragten Personen an, innerhalb einer medizinischen Einrichtung betroffen gewesen zu sein. $1 \%$ der Stichprobe bejahte zudem, in einer medizinischen Institution körperliche Gewalt erlebt zu haben (Witt, Rassenhofer et al., 2019). Wenig später wurde eine umfangreiche und differenzierte repräsentative Studie zu Kindesmisshandlung im institutionellen Kontext in Deutschland durchgeführt, darunter auch im medizinischen Bereich, deren Ergebnisse in Tabelle 1 zu sehen sind (Clemens et al., 2019; Hoffmann, Clemens, König, Brähler \& Fegert, 2020). Von den im Rahmen dieser Erhebung befragten 2516 Personen im Alter von 18 bis 90 Jahren hatten 544 in ihrer Kindheit einen Aufenthalt in einer medizinischen Einrichtung, also z.B. einer Allgemeinklinik oder einer Kinderklinik (Clemens et al., 2019). $19 \%$ dieser Personen, $17 \%$ der Frauen und 22\% der Männer, waren dabei von mindestens einer Misshandlungsform betroffen. Sie hatten vor allem Vernachlässigung erlebt, die sich darin äußerte, dass die Betroffenen Pflege und Versorgung als nicht ausreichend oder nicht gut erlebt, oder das Gefühl gehabt hatten, dass es niemanden interessierte, wie es ihnen ging. Zudem hatte jeweils jede zehnte Person, die sich in ihrer Kindheit in einer medizinischen Einrichtung aufgehalten hatte, körperliche Gewalt in Form von Schlägen bzw. einem rauen Umgang bei Behandlungen oder Zwangsmaßnahmen oder emotionale Gewalt durch Erniedrigung, Beleidigung, Einschüchterung und Drohungen oder Weitergabe vertraulicher Informationen hinter ihrem Rücken erlebt. Sexueller Missbrauch war innerhalb medizinischer Einrichtungen seltener vorgefallen, wobei sowohl Übergriffe ohne als auch mit Penetration genannt wurden. Bis auf eine signifikant höhere Rate emotionaler Misshandlung bei Befragten ab einem Alter von 60 Jahren gab es keine signifikanten Unterschiede zwischen den befragten Kohorten. Es ergeben sich aus dieser Studie keine Hinweise darauf, dass sich die Prävalenzen anderer Formen von Kindesmisshandlung in medizinischen Einrichtungen im Verlauf der letzten Jahrzehnte signifikant verändert haben. In Bezug auf die Gesamtstichprobe - also unter Einschluss aller rund 2500 Personen, unabhängig davon, ob sie in ihrer Kindheit eine medizinische Einrichtung aufgesucht hatten - sind die von Clemens und Kollegen (2019) berichteten Prävalenzen körperlicher, emotionaler und sexualisierter Gewalt sowie Vernachlässigung zudem höher, als die Ergebnisse von Witt, Rassenhofer und Kollegen (2019) vermuten lassen. 
M. Rassenhofer et al., Übergriffe durch medizinisch-therapeutisches Personal

221

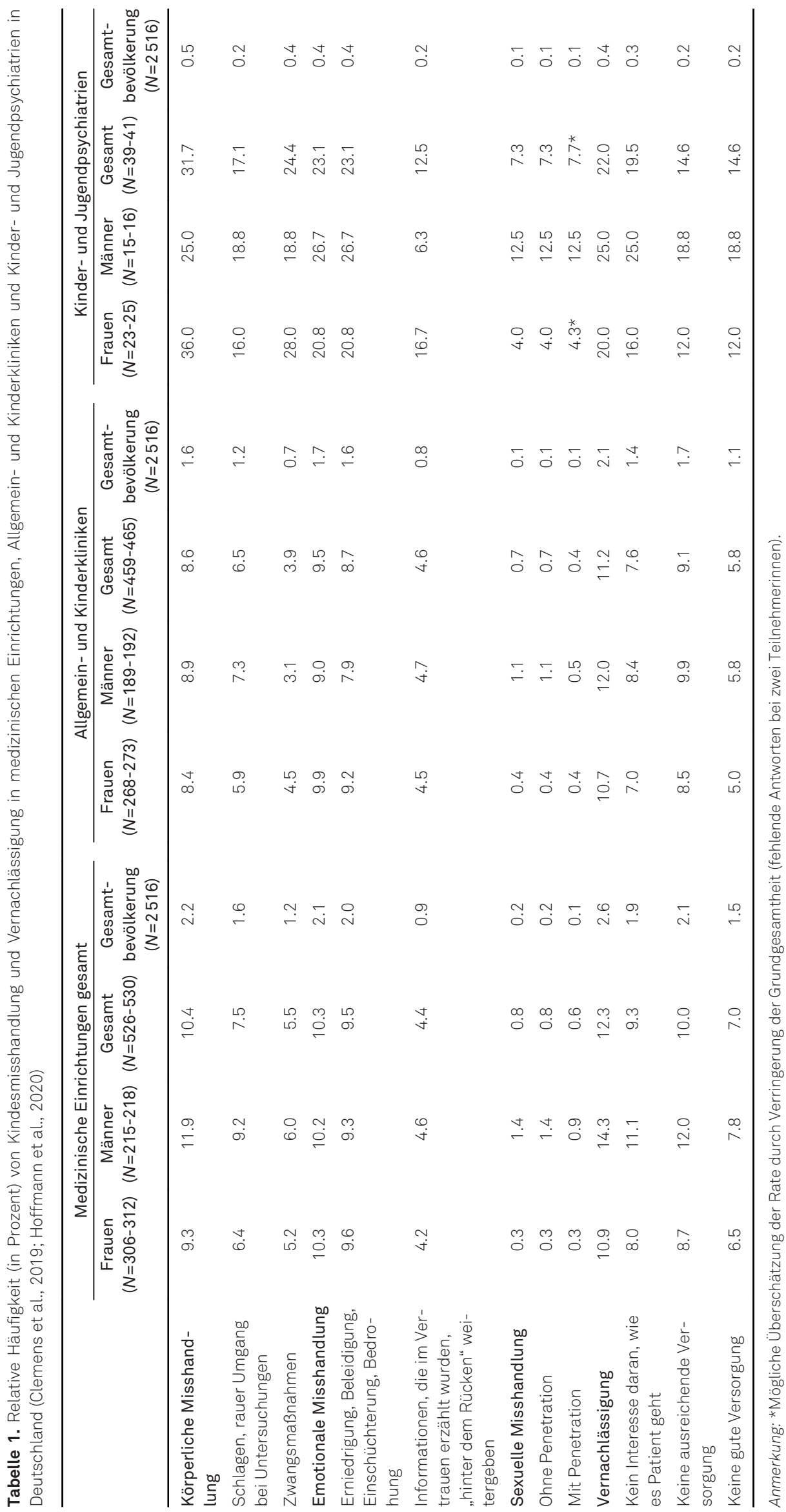

(c) 2021 The Author(s). Distributed as a Hogrefe OpenMind article

Kindheit und Entwicklung (2021), 30 (4), 218-226

under the license CC BY-NC-ND 4.0 (https://creativecommons.org/licenses/by-nc-nd/4.0) 


\section{Häufigkeit von Übergriffen in Allgemein- und Kinderkliniken in Deutschland}

Hoffmann und Kolleg_innen (2020) differenzierten die von Clemens und Kolleg_innen (2019) erstmals beschriebene Stichprobe weiter nach Kinder- und Allgemeinkliniken sowie Kinder- und Jugendpsychiatrien. Dabei waren die Prävalenzen der verschiedenen Formen von Kindesmisshandlung in Allgemein- und Kinderkliniken etwas geringer als in medizinischen Einrichtungen insgesamt (siehe Tabelle 1). Von den 474 Personen, die in ihrer Kindheit stationär in einer Klinik waren, erlebten $17.3 \%$ mindestens eine Form von Misshandlung oder Vernachlässigung während ihres Aufenthalts, wovon etwa die Hälfte von mehr als einem Übergriff berichtete (Hoffmann et al., 2020). Am häufigsten waren Erlebnisse von Vernachlässigung, emotionaler und körperlicher Gewalt. Bei etwa zwei Dritteln der Berichte von körperlichen Misshandlungen in Kliniken und jeweils etwa 85\% der Fälle emotionaler Gewalt und Vernachlässigung waren Frauen als Täterinnen beteiligt. Männer waren in etwa einem Drittel der Fälle emotionaler Gewalt und Vernachlässigung als Täter beteiligt und bei $55 \%$ der körperlichen Misshandlungen. Sexualisierte Gewalt ging ausschließlich von männlichen Tätern aus. Anders als in der Allgemeinbevölkerung waren Männer bei Krankenhausaufenthalten in der Kindheit häufiger von sexualisierter Gewalt betroffen gewesen als Frauen, sowohl bei Übergriffen ohne als auch mit Penetration. Auch Vernachlässigung und körperliche Misshandlung hatten Männer häufiger erlebt als Frauen. Frauen waren hingegen häufiger von Zwangsmaßnahmen und emotionaler Misshandlung betroffen (siehe Tabelle 1).

\section{Häufigkeit von Übergriffen in kinder- und jugendpsychiatrischen Kliniken}

\section{Internationale Daten}

Die ersten Daten zur Häufigkeit von Misshandlung und Vernachlässigung in kinder- und jugendpsychiatrischen Kliniken stammen aus einer Befragung zur Behandlungszufriedenheit von Patient_innen und ihren Eltern einer Kinder- und Jugendpsychiatrie in Pennsylvania (Kaplan, Busner, Chibnall \& Kang, 2001). 21\% der Eltern und 28\% der Kinder berichteten von verbaler sowie $5 \%$ der Eltern und $10 \%$ der Kinder von körperlicher Gewalt durch das Personal. Weniger als 1\% der Eltern und 1\% der Kinder gab an, dass es während des Aufenthalts zu einem sexuellen Übergriff durch Klinikpersonal gekommen war. El- tern und Kinder, die von Gewalterfahrungen während des Klinikaufenthalts berichtet hatten, waren signifikant weniger zufrieden mit dem Personal und wollten die Kinderund Jugendpsychiatrie weniger wahrscheinlich erneut aufsuchen; der wahrgenommene Behandlungserfolg unterschied sich jedoch nicht zu Familien, die nicht mit Übergriffen durch das Personal konfrontiert gewesen waren.

Während die Prävalenz von Misshandlung und Vernachlässigung in Kinder- und Jugendpsychiatrien kaum erforscht ist, wurden in den letzten Jahren vermehrt Studien zur Häufigkeit der Anwendung von Zwangsmaßnahmen veröffentlicht. Laut einem systematischen Literaturreview von De Hert, Dirix, Demunter und Correll (2011), welcher die Ergebnisse von Studien aus den USA, Australien und Finnland zusammenfasst, werden im Mittel 26\% der Patient_innen in Kinder- und Jugendpsychiatrien mindestens einmal während ihres Aufenthalts isoliert und $29 \%$ fixiert. Dabei ergaben sich große Unterschiede zwischen den verglichenen Studien mit Raten von Isolierungen bei 8.5-61\% der Patient_innen und Fixierungen bei 7.6-47\%. Auch die Häufigkeit von Zwangsmaßnahmen im Laufe eines Behandlungsjahres schwankte stark zwischen den Kliniken. Im Schnitt wurden an 67/1000 Behandlungstagen Patient_innen isoliert (Range: 14.592/1000) und an 42.7/1000 Behandlungstagen Patient_innen fixiert (Range: 21.2-56/1000).

\section{Daten aus dem deutschsprachigen Raum}

\section{Zwangsmaßnahmen}

Seit Inkrafttreten des Psychisch-Kranken-Hilfe-Gesetzes (PsychKHG) im Jahr 2015 sind psychiatrische Kliniken in Baden-Württemberg gesetzlich dazu verpflichtet, Unterbringungs- und Zwangsmaßnahmen genau zu dokumentieren, sodass umfangreiche Einblicke in die Praxis der Durchführung von Zwangsmaßnahmen gewonnen werden können (Flammer \& Steinert, 2019). Eine erste Auswertung hierdurch gesammelter Daten von 32 psychiatrischen, psychosomatischen und kinder- und jugendpsychiatrischen Kliniken in Baden-Württemberg durch Flammer und Steinert (2019) ergab, dass pro Klinik durchschnittlich $6.7 \%$ der Patient_innen innerhalb eines Jahres von einer Zwangsmaßnahme betroffen waren. 3.7\% der Patient_innen wurden fixiert, $1.8 \%$ isoliert, $1.1 \%$ der Patient_innen fixiert und isoliert und $0.6 \%$ der Patient_innen medikamentös zwangsbehandelt. Am häufigsten von Zwangsmaßnahmen betroffen waren dabei Patient_innen mit Diagnosen der Störungskategorien FO (organischpsychische Störungen), F2 (schizophrene und wahnhafte Störungen), F6 (Persönlichkeitsstörungen), F7 (Intelligenzminderung) sowie F8 (Entwicklungsstörungen). 
Auch schon vor Inkrafttreten des PsychKHG wurde der Einsatz von Zwangsmaßnahmen in Kliniken dokumentiert und mithilfe von Patientendatenbanken ausgewertet. In einer kinder- und jugendpsychiatrischen Klinik in der Schweiz erfuhren im Zeitraum 2002/2003 17\% der Patient_innen Zwangsmaßnahmen, davon 87\% Isolierungen, $14 \%$ Zwangsmedikation und 2\% Fixierungen (NitschkeJanssen \& Branik, 2006). Durchschnittlich wurden an 8.28/1000 Behandlungstagen Zwangsmaßnahmen durchgeführt. $42 \%$ der von Zwangsmaßnahmen betroffenen Patient_innen erlebten nur eine Zwangsmaßnahme. Mädchen waren fast doppelt so häufig von Zwangsmaßnahmen betroffen wie Jungen und Jugendliche fast doppelt so häufig wie Kinder. Am häufigsten waren unter den Patient_innen, bei denen Zwangsmaßnahmen angewendet wurden, Diagnosen der Störungskategorien F9 (Verhaltens- und emotionale Störungen mit Beginn in der Kindheit und Jugend, $48 \%)$, F6 (28\%), F2 (11\%) und F4 (neurotische Belastungs- und somatoforme Störungen, $7 \%)$ vergeben worden.

In gleicher Weise analysierten Fetzer, Steinert, Metzger und Fegert (2006) im Zeitraum 1999-2004 sowie Closset (2016) im Zeitraum 2005-2011 eine Datenbank zu Zwangsmaßnahmen in der kinder- und jugendpsychiatrischen Klinik im Zentrum für Psychiatrie (ZfP) Weissenau. Im ersten Untersuchungszeitraum waren $9.2 \%$ der behandelten Kinder und Jugendlichen von Zwangsmaßnahmen betroffen, im zweiten Untersuchungszeitraum $12 \%$. $53.4 \%$ der Patient_innen erlebten nur eine Zwangsmaßnahme, im Durchschnitt waren es 3.4. Größtenteils (knapp 90\%) handelte es bei den Zwangsmaßnahmen um Isolierungen. Fixierungen und Zwangsmedikation lagen jeweils im einstelligen Prozentbereich. Insgesamt hatten in beiden Untersuchungszeiträumen die meisten Patient_innen, bei denen Zwangsmaßnahmen angewendet wurden, eine F9-Diagnose (52.8\%). Wie in der Studie von Nitschke-Janssen und Branik waren ältere Patient_innen häufiger und Mädchen beinahe doppelt so vielen Zwangsmaßnahmen ausgesetzt wie Jungen. Beim Vergleich von Patient_innen mit und ohne Zwangsmaßnahmen ergab sich jedoch ein höherer Anteil männlicher Patienten unter Patient_innen mit Zwangsmaßnahmen sowie kein signifikanter Unterschied in der Beurteilung des Therapieerfolgs.

Ähnlich der Befunde von Swahnberg und Kolleg_innen (2007) zum Zusammenhang früherer Misshandlungserfahrungen und der Wahrscheinlichkeit, Misshandlung bei medizinischen Behandlungen zu erleben, scheint es auch eine Häufung des Einsatzes von Zwangsmaßnahmen bei Patient_innen mit anamnestisch bekannten Misshandlungserfahrungen zu geben. Bei einer Patientendatenbankanalyse einer allgemeinpsychiatrischen Klinik zeigte sich, dass Patient_innen, die besonders häufig oder lange fixiert oder isoliert wurden, signifikant häufiger von Misshandlungserfahrungen in der Kindheit berichtet hatten (Hammer, Springer, Beck, Menditto \& Coleman, 2011). Auch in der Studie von Nitschke-Janssen und Branik (2006) hatten $75 \%$ der Kinder, die von einer Zwangsmaßnahme betroffen waren, von Gewalterfahrungen innerhalb oder außerhalb der Familie berichtet. Hingegen zeigten sich beim Vergleich von Patien_innen mit Zwangsmaßnahmen (44.9\%) und ohne Zwangsmaßnahmen $(40 \%)$ keine signifikanten Unterschiede in der Häufigkeit von Gewalterfahrungen vor dem Aufenthalt im ZfP Weissenau (Closset, 2016).

\section{Misshandlung und Vernachlässigung}

Gewalterfahrungen in deutschen Psychiatrien stellen keine Seltenheit dar, ihre Häufigkeit wurde bislang jedoch kaum systematisch oder spezifisch für den Bereich der Kinder- und Jugendpsychiatrie untersucht. Die einzige uns bekannte Studie, welche die Häufigkeit von Misshandlung, Vernachlässigung und Zwangsmaßnahmen in Kinder- und Jugendpsychiatrien repräsentativ erhoben hat, ist die bereits beschriebene Untersuchung von Clemens und Kolleg_innen (2019), deren Ergebnisse Hoffmann und Kolleg_innen (2020) weiter nach Kliniktyp differenzierten. Unter den insgesamt ca. 2500 befragten Personen waren 46 stationär in einer Kinder- und Jugendpsychiatrie. Von diesen berichteten $33.3 \%$ von mindestens einer Form von Misshandlung oder Vernachlässigung während ihres Aufenthalts. Diese Rate war nur etwas geringer, wenn Zwangsmaßnahmen nicht als Misshandlung gezählt wurden. Etwa ein Drittel berichtete von Erlebnissen körperlicher Gewalt, und jeweils knapp ein Viertel gab an, emotionale Gewalt oder Vernachlässigung in einer Kinder- und Jugendpsychiatrie erfahren zu haben (siehe Tabelle 1). Eine Frau und zwei Männer berichteten von sexuellem Missbrauch sowohl ohne als auch mit Penetration während ihres Aufenthalts in der Kinder- und Jugendpsychiatrie. Neben sexualisierter Gewalt waren Männer häufiger von Schlägen und einem rauen Umgang während Untersuchungen sowie Vernachlässigung betroffen als Frauen. Frauen waren hingegen häufiger von Zwangsmaßnahmen betroffen. Bei den berichteten Fällen körperlicher Gewalt und Vernachlässigung waren in drei Vierteln der Fälle Männer und in etwa 55\% der Fälle Frauen als Täter_in beteiligt. Emotionale Gewalt ging in zwei Dritteln der Fälle von Männern sowie in $44 \%$ von Frauen und sexualisierte Gewalt ausschließlich von Männern aus. Durch den großen Altersumfang der Stichprobe konnte auch die Veränderung der Häufigkeit von Misshandlungen und des Einsatzes von Zwangsmaßnahmen vor und nach der Veröffentlichung des Berichts über die Lage der Psychiatrie in der Bundesrepublik Deutschland (Psychiatrie-Enquête) verglichen werden. Dieser 1975 
nach einer Beauftragung durch den Bundestag veröffentlichte Bericht machte auf die Rechte psychisch Kranker und die Verpflichtung zum ethischen Umgang mit ihnen aufmerksam. Tatsächlich waren die Raten der Berichte von Schlägen und einem rauen Umgang während der Behandlung bzw. von Zwangsmaßnahmen unter ehemaligen Patient_innen der Kinder- und Jugendpsychiatrie, die vor dem Jahr 1975 ihr 18. Lebensjahr erreichten (42.9\% bzw. 28.6\%), höher als unter ehemaligen Patient_innen, die nach dem Jahr 1975 ihr 18. Lebensjahr erreichten (11.8\% bzw. 23.5\%). Die geringe Stichprobengröße ließ jedoch keine statistischen Vergleiche zu. Insgesamt ist die Studienlage zur Häufigkeit von Misshandlung und Vernachlässigung im Bereich der Kinder- und Jugendpsychiatrie zu klein, um genaue Prävalenzen ableiten zu können. Die in den hier dargestellten Studien berichteten Häufigkeiten sind jedoch ein Hinweis darauf, dass Gewalt in kinder- und jugendpsychiatrischen Kliniken ein weit verbreitetes Problem darstellt und nicht nur in Einzelfällen auftritt.

\section{Häufigkeit von Übergriffen im Rahmen ambulanter Psychotherapie}

Für den ambulanten psychotherapeutischen Bereich gibt es kaum belastbare Zahlen zur Häufigkeit von Übergriffen auf Kinder und Jugendliche. Noyon (2011) fasste die wenigen Forschungsergebnisse zur Häufigkeit sexueller Übergriffe auf Patient_innen durch Psychotherapeut_innen zusammen, die sich jedoch nicht spezifisch auf den Kinder- und Jugendbereich beziehen. Demnach werden jährlich etwa 300 Fälle sexuellen Missbrauchs durch Psychotherapeut_innen mit Kassensitz gemeldet, und 6\% der Psychotherapeut_innen in Deutschland begehen im Laufe ihres Berufslebens mindestens einen sexuellen Übergriff. Dies ist vergleichbar mit internationalen Studien (Franke \& Riecher-Rössler, 2011). Eichenberg, BeckerFischer und Fischer (2010) analysierten die Angaben von 77 Patient_innen, deren Therapeut_innen sexuelle Annäherungen unternommen hatten, hierunter zehn Patient_innen, die zum Zeitpunkt des sexuellen Kontakts minderjährig waren. Sie konnten zeigen, dass es keine Unterschiede in der Häufigkeit sexuell grenzverletzenden Verhaltens zwischen verschiedenen Therapieströmungen und psychotherapeutisch tätigen Berufsgruppen gibt und dass die Taten bei der Mehrheit der Befragten negative Konsequenzen sowie Verschlechterung der Ausgangssymptomatik zur Folge hatten. Wie auch in einer Studie von Stuhler, Kontny, Schleu und Strauß (2019) handelte es sich zudem bei den Betroffenen meist um weibliche
Patientinnen und bei den Therapeut_innen vor allem um Männer. Auch in den bereits genannten Analysen zur Begleitforschung der UBSKM konnten unter den Betroffenen im medizinisch-therapeutischen Bereich Personengruppen identifiziert werden, die im Rahmen einer Psychotherapie sexuell missbraucht wurden (Fegert et al., 2013). Unter anderem hatten sich dort Personen gemeldet, die sich zur Aufarbeitung eines Missbrauchs in Therapie begeben hatten und dort erneut sexuell missbraucht wurden, was meist einen großen Vertrauensverlust in therapeutische Hilfe im Allgemeinen zur Folge hatte. Die genannten Befunde verdeutlichen zwar, dass sich sexualisierte Gewalt im Rahmen psychotherapeutischer Behandlungen nicht nur auf Einzelfälle beschränkt, sind jedoch weder repräsentativ für die Bevölkerung, noch lassen sie verallgemeinernde Rückschlüsse auf den kinderund jugendpsychotherapeutischen Bereich zu.

\section{Limitationen}

Der in diesem Artikel dargestellte Überblick über den aktuellen Forschungsstand zur Prävalenz von Übergriffen durch Angehörige der Heil- und Pflegeberufe erhebt nicht den Anspruch eines systematischen Literaturreviews, sondern zielt vielmehr darauf ab, einen möglichst umfassenden Einblick zu geben, um eine Einordnung der Häufigkeit von Übergriffen auf Kinder und Jugendliche im Rahmen medizinisch-therapeutischer Behandlungen zu ermöglichen. Die hierfür eingeschlossenen Artikel unterscheiden sich daher stark in ihrer methodischen Qualität. Es wurden sowohl Prävalenzermittlungen aus Metaanalysen, repräsentativen Bevölkerungsstichproben, Krankenhausdatenbanken sowie willkürlichen Stichproben berücksichtigt. Die Daten umfassen sowohl unmittelbar als auch retrospektiv - und somit Gedächtnisphänomenen unterliegend - erhobene Erfahrungen von Betroffenen, Bezugspersonen und medizinischem Personal. Zudem wurden aufgrund der mangelnden Studienlage teilweise Untersuchungen zu Häufigkeiten von Übergriffen auf erwachsene Patient_innen eingeschlossen und berichtet. Die Untersuchungen zu betroffenen Kindern und Jugendlichen unterscheiden nicht hinsichtlich verschiedener Alters- und Entwicklungsstufen zum Zeitpunkt der Übergriffe. Dies sollte in zukünftigen Studien berücksichtigt werden. Die wiedergegebenen Prävalenzen von Zwangsmaßnahmen oder auch die mithilfe des NorAQ ermittelten Prävalenzen leichter Übergriffe im Rahmen medizinischer Behandlungen lassen keine Rückschlüsse auf die durch diese Erfahrungen erlebte Belastung bei den Betroffenen zu (vgl. jedoch Christiansen \& Barke, 2021). 
Insgesamt sind die im vorliegenden Artikel berichteten Prävalenzen nicht direkt miteinander vergleichbar und lassen keine verallgemeinernden Rückschlüsse auf medizinische Einrichtungen zu. Sie verdeutlichen jedoch übergreifend das Vorhandensein der Problematik sowie die Notwendigkeit weiterer epidemiologischer Studien.

\section{Zusammenfassung und Fazit}

Der vorliegende Artikel weist auf eine hochgradig alarmierende Häufigkeit von Übergriffen auf Kinder und Jugendliche durch pflegendes und medizinisch-therapeutisches Personal hin. In Deutschland war im Rahmen der Behandlung in medizinischen Einrichtungen etwa ein Fünftel der Bevölkerung von mindestens einer Form von Übergriffen in der Kindheit und Jugend durch das Einrichtungspersonal betroffen. Während eines Aufenthalts in einer Allgemein- oder Kinderklinik bzw. in einer Kinder- und Jugendpsychiatrie erlebten ebenfalls rund ein Fünftel der befragten Deutschen mindestens eine Form von Gewalt oder Vernachlässigung durch das Pflegepersonal. Diese Befunde stammen aus der bislang einzigen uns bekannten repräsentativen Erhebung zur Prävalenz von Kindesmisshandlung in klinischen Einrichtungen in Deutschland (Clemens et al., 2019; Hoffmann et al., 2020). Vergleiche mit Ergebnissen anderer Studien lassen offen, inwiefern sie die tatsächliche Verbreitung von Gewalt in Institutionen widerspiegeln. In medizinischen Einrichtungen wurden bisher geringere Raten an Kindesmisshandlung und -vernachlässigung erfasst, die meist im einstelligen Prozentbereich liegen (Lukasse et al., 2015; Swahnberg et al., 2012; Witt, Rassenhofer et al., 2019). Die von Hoffmann und Kolleg_innen (2020) berichteten Häufigkeiten von Misshandlung, Vernachlässigung und Zwangsmaßnahmen in Kinder- und Jugendpsychiatrien sind ebenfalls höher als bislang durch andere nationale und internationale Studien bekannt (Closset, 2016; Fetzer et al., 2006; Nitschke-Janssen \& Branik, 2006). Prädiktoren für das Erleben von Misshandlung, Missbrauch und Vernachlässigung im medizinisch-therapeutischen Bereich scheinen unter anderem Geschlecht, Alter, spezifische psychiatrische Diagnosen sowie bisherige Gewalterfahrungen der Betroffenen zu sein. Da Personen, die bereits Misshandlung und Vernachlässigung erfahren haben, durch Heil- und Pflegepersonal besonderen Schutz erfahren sollten, jedoch anscheinend einer höheren Gefährdung für Übergriffe ausgesetzt sind, müssen genauere Untersuchungen durchgeführt werden, um Möglichkeiten $\mathrm{zu}$ finden, diesen Risikofaktor $\mathrm{zu}$ minimieren. Die im Rahmen dieses Artikels angedeuteten Folgen von Übergriffen im klinischen Setting, unter anderem der Vertrau- ensverlust in medizinisch-therapeutisches Personal und die Verschlechterung psychischer Symptome, geben Hinweise darauf, dass auch in diesem Bereich Missbrauch, Misshandlung und Vernachlässigung mit schweren Folgen für die Betroffenen einhergehen. Es wird ersichtlich, dass Studien durchgeführt werden müssen, um mehr Wissen über Prävalenzen und Präventionsmöglichkeiten in medizinisch-therapeutischen Institutionen zu gewinnen.

\section{Literatur}

Aguilera-Serrano, C., Guzman-Parra, J., Garcia-Sanchez, J. A., Moreno-Küstner, B. \& Mayoral-Cleries, F. (2018). Variables associated with the subjective experience of coercive measures in psychiatric inpatients: A systematic review. Canadian journal of psychiatry, 63, 129-144. https://doi.org/10.1177/0706743717 738491

Christiansen, H. \& Barke, A. (2021). Gewalterfahrungen und Traumafolgestörungen bei Kindern und Jugendlichen. Kindheit und Entwicklung, 30, 137-143.

Clemens, V., Hoffmann, U., König, E., Sachser, C., Brähler, E. \& Fegert, J. M. (2019). Child maltreatment by nursing staff and caregivers in German institutions: a population-representative analysis. Child Abuse and Neglect, 95, Art. 104046. https://doi. org/10.1016/j.chiabu.2019.104046

Closset, J. C. (2016). Zwangsmaßnahmen bei Kindern und Jugendlichen: Eine Analyse von Entwicklung, Zeitverlauf und Diagnoseverteilung von Zwangsmaßnahmen, sowie Untersuchung von Patientencharakteristika (Unveröffentlichte Dissertation). Universität Ulm. https://oparu.uni-ulm.de/xmlui/bitstream/hand le/123456789/3832/vts_9950_15166.pdf?sequence=1\&isAllo wed $=y$

De Hert, M., Dirix, N., Demunter, H. \& Correll, C. U. (2011). Prevalence and correlates of seclusion and restraint use in children and adolescents: A systematic review. European Child and Adolescent Psychiatry, 20, 221 -230. https://doi.org/10.1007/ s00787-011-0160-x

Eichenberg, C., Becker-Fischer, M. \& Fischer, G. (2010). Sexual assaults in therapeutic relationships: prevalence, risk factors and consequences. Health, 2, 1018-1026. https://doi.org/10. 4236/health.2010.29150

Fegert, J. M., Bergmann, C., Spröber, N. \& Rassenhofer, M. (2013). Belastungen durch sexuellen Missbrauch und medizinische und therapeutische Behandlung. Nervenheilkunde, 32, $827-$ 833. https://doi.org/10.1055/s-0038-1628558

Fetzer, A. E., Steinert, T., Metzger, W. \& Fegert, J. M. (2006). Eine prospektive Untersuchung von Zwangsmaßnahmen in der stationären Kinder- und Jugendpsychiatrie. Praxis der Kinderpsychologie und Kinderpsychiatrie, 55, 754-766. http://hdl.handle. net/20.500.11780/2949

Flammer, E. \& Steinert, T. (2019). Das Fallregister für Zwangsmaßnahmen nach dem baden-württembergischen PsychischKranken-Hilfe-Gesetz: Konzeption und erste Auswertungen. Psychiatrische Praxis, 46, 82-89. https://doi.org/10.1055/a0665-6728

Franke, I. \& Riecher-Rössler, A. (2011). Missbrauch in therapeutischen Beziehungen. Der Nervenarzt, 82, 1145-1150. https:// doi.org/10.1007/s00115-010-3211-5 
Hammer, J. H., Springer, J., Beck, N. C., Menditto, A. \& Coleman, J. (2011). The relationship between seclusion and restraint use and childhood abuse among psychiatric inpatients. Journal of Interpersonal Violence, 26, 567-579. https://doi.org/10.1177/ 0886260510363419

Hoffmann, U., Clemens, V., König, E., Brähler, E. \& Fegert, J. M. (2020). Violence against children and adolescents by nursing staff: Prevalence rates and implications for practice. Child and Adolescent Psychiatry and Mental Health, 14, Art. 43. https:// doi.org/10.1186/s13034-020-00350-6

Hoffmann, U., Fegert, J. M., Jud, A., Clemens, V. \& Rassenhofer, M. (2021). Schutz vor Gewalt und Übergriffen in medizinischen Institutionen - Ursachen, Häufigkeiten und Implikationen für die Praxis. Praxis der Kinderpsychologie und Kinderpsychiatrie, 70, 64-83. https://doi.org/10.13109/prkk.2021.70.1.64

Hoffmann, U., Fegert, J. M., König, E., Maier, A. \& Herberhold, M. (2021). Entwicklung von Schutzkonzepten gegen (sexuelle) Gewalt im medizinisch-therapeituischen Bereich. Kindheit und Entwicklung, 30, 227-235.

Iffland, B., Brähler, E., Neuner, F., Häuser, W. \& Glaesmer, H. (2013). Frequency of child maltreatment in a representative sample of the German population. BMC Public Health, 13, Art. 980. https://doi.org/10.1186/1471-2458-13-980

Jones, L., Bellis, M. A., Wood, S., Hughes, K., McCoy, E., Eckley et al. (2012). Prevalence and risk of violence against children with disabilities: A systematic review and meta-analysis of observational studies. The Lancet, 380, 899-907. https://doi.org/10. 1016/S0140-6736(12)60692-8

Kaplan, S., Busner, J., Chibnall, J. \& Kang, G. (2001). Consumer satisfaction at a child and adolescent state psychiatric hospital. Psychiatric Services, 52, 202 - 206. https://doi.org/10.1176/ appi.ps.52.2.202

Katz, C. C., Courtney, M.E. \& Novotny, E. (2017). Pre-foster care maltreatment class as a predictor of maltreatment in foster care. Child and Adolescent Social Work Journal, 34, 35-49. https://doi.org/10.1007/s10560-016-0476-y

Lukasse, M., Schroll, A. M., Karro, H., Schei, B., Steingrimsdottir, T., Van Parys et al. (2015). Prevalence of experienced abuse in healthcare and associated obstetric characteristics in six European countries. Acta Obstetricia et Gynecologica Scandinavica, 94, 508-517. https://doi.org/10.1111/aogs.12593

Nitschke-Janssen M. \& Branik, E. (2006). Einflussfaktoren auf den Einsatz von Zwangsmaßnahmen - Eine retrospektive Auswertung in einer Schweizer kinder- und jugendpsychiatrischen Versorgungsklinik. Praxis der Kinderpsychologie und Kinderpsychiatrie, 55, 255 -270. http://hdl.handle.net/20.500.11780/ 2907

Noyon, A. (2011). Aspekte der Klinischen Psychologie: Sexueller Missbrauch in Beratungskontexten. In M. Baldus \& R. Utz (Hrsg.), Sexueller Missbrauch in pädagogischen Kontexten (S. 159 -176). Wiesbaden: VS Verlag für Sozialwissenschaften. https://doi.org/10.1007/978-3-531-93353-5_8

Rassenhofer, M. (2020). Die Folgen von Kindesmisshandlung und ihre psychotherapeutische Behandlung: Eine praxisorientierte Übersicht. Zeitschrift für Psychiatrie, Psychologie und Psychotherapie, 68, 5-15. https://doi.org/10.1024/1661-4747/a000400

Roy, C., Castonguay, A., Fortin, M., Drolet, C., Franche-Choquette, G., Dumais, A. et al. (2021). The use of restraint and seclusion in residential treatment care for youth: A systematic review of related factors and interventions. Trauma, Violence, and Abuse, 22, 318 -338. https://doi.org/10.1177/1524838019843196

Schulte, K. L., Szota, K. \& Christiansen, H. (2021). Die Entwicklung von Sexualität bei Kindern und Jugendlichen mit sexuellen Gewalterfahrungen. Eine systematische Übersicht über Auswir- kungen auf die Sexualität. Kindheit und Entwicklung, 30, 172 182.

Spröber-Kolb, N., Rassenhofer, M., Allroggen, M., Plener, P. L., Kölch, M. \& Fegert, J. M. (2017). Research on child sexual abuse in institutions in German-speaking countries: A summary. In A. Rus, S. Parris \& E. Stativa (Eds.), Child maltreatment in residential care (pp. $179-197)$. Cham: Springer. https://doi.org/10. 1007/978-3-319-57990-0_9

Spröber, N., Schneider, T., Rassenhofer, M., Seitz, A., Liebhardt, H., König, L. et al. (2014). Child sexual abuse in religiously affiliated and secular institutions: A retrospective descriptive analysis of data provided by victims in a government-sponsored reappraisal program in Germany. BMC Public Health, 14, Art. 282. https://doi.org/10.1186/1471-2458-14-282

Stoltenborgh, M., van IJzendoorn, M. H., Euser, E. M. \& Bakermans-Kranenburg, M. J. (2011). A global perspective on child sexual abuse: Meta-Analysis of prevalence around the world. Child Maltreatment, 16, 79-101. https://doi.org/10.1177/ 1077559511403920

Stuhler, H., Kontny, L., Schleu, A. \& Strauß, B. (2019). Von schleichenden Grenzverletzungen zu sexuellem Missbrauch in Psychotherapien. Psychotherapeut, 64, 470 - 475. https://doi.org/ 10.1007/s00278-019-00386-w

Swahnberg, K., Davidsson-Simmons, J., Hearn, J. \& Wijma, B. (2012). Men's experiences of emotional, physical, and sexual abuse and abuse in health care: a cross-sectional study of a Swedish random male population sample. Scandinavian journal of public health, 40, 191-202. https://doi.org/10.1177/ 1403494811425711

Swahnberg, K., Schei, B., Hilden, M., Halmesmäki, E., Sidenius, K., Steingrimsdottir, T. et al. (2007). Patients' experiences of abuse in health care: A Nordic study on prevalence and associated factors in gynecological patients. Acta Obstetricia et Gynecologica Scandinavica, 86, 349-356. https://doi.org/10.1080/ 00016340601185368

Swahnberg, I. M. K. \& Wijma, B. (2003). The NorVold Abuse Questionnaire (NorAQ) Validation of new measures of emotional, physical, and sexual abuse, and abuse in the health care system among women. The European Journal of Public Health, 13, 361 - 366. https://doi.org/10.1093/eurpub/13.4.361

Witt, A., Brown, R. C., Plener, P. L., Brähler, E. \& Fegert, J. M. (2017). Child maltreatment in Germany: Prevalence rates in the general population. Child and Adolescent Psychiatry and Mental Health, 11, Art. 47. https://doi.org/10.1186/s13034-017-0185-0

Witt, A., Glaesmer, H., Jud, A., Plener, P. L., Brähler, E., Brown, R. C. et al. (2018). Trends in child maltreatment in Germany: Comparison of two representative population-based studies. Child and adolescent psychiatry and mental health, 12, Art. 24. https:// doi.org/10.1186/s13034-018-0232-5

Witt, A., Rassenhofer, M., Allroggen, M., Brähler, E., Plener, P. L. \& Fegert, J. M. (2019). The prevalence of sexual abuse in institutions: Results from a representative population-based sample in Germany. Sexual Abuse, 31, 643-661. https://doi.org/10. $1177 / 1079063218759323$

\section{Jun.-Prof. Dr. Miriam Rassenhofer}

Klinik für Kinder- und Jugendpsychiatrie/Psychotherapie UIm Steinhövelstraße 5

89075 Ulm

miriam.rassenhofer@uniklinik-ulm.de 